\title{
WRKY Transcription Factors Shared by BTH-Induced Resistance and NPR1-Mediated Acquired Resistance Improve Broad-Spectrum Disease Resistance in Wheat
}

\author{
Huanpeng Li, ${ }^{1}$ Jiaojiao Wu, ${ }^{1}$ Xiaofeng Shang, ${ }^{1}$ Miaomiao Geng, ${ }^{3}$ Jing Gao, ${ }^{1}$ Shuqing Zhao, ${ }^{1}$ Xiumei Yu, \\ Daqun Liu, ${ }^{1}$ Zhensheng Kang, ${ }^{2}$ Xiaojie Wang, ${ }^{2,+}$ and Xiaodong Wang ${ }^{1,+}$ \\ ${ }^{1}$ State Key Laboratory of North China Crop Improvement and Regulation, College of Plant Protection, Technological Innovation \\ Center for Biological Control of Crop Diseases and Insect Pests of Hebei Province, Hebei Agricultural University, Baoding, \\ Hebei 071000 , P. R. China \\ ${ }^{2}$ State Key Laboratory of Crop Stress Biology for Arid Areas, College of Plant Protection, Northwest Agriculture and Forestry \\ University, Yangling, Shaanxi 712100, P. R. China \\ ${ }^{3}$ College of Agronomy, Hebei Agricultural University \\ ${ }^{4}$ College of Life Science, Hebei Agricultural University
}

Accepted 9 December 2019.

In Arabidopsis, both pathogen invasion and benzothiadiazole (BTH) treatment activate the nonexpresser of pathogenesisrelated genes 1 (NPR1)-mediated systemic acquired resistance, which provides broad-spectrum disease resistance to secondary pathogen infection. However, the BTH-induced resistance in Triticeae crops of wheat and barley seems to be accomplished through an NPRI-independent pathway. In the current investigation, we applied transcriptome analysis on barley transgenic lines overexpressing wheat wNPR1 (wNPR1-OE) and knocking down barley HvNPRI (HvNPRI-Kd) to reveal the role of NPR1 during the BTH-induced resistance. Most of the previously designated barley chemical-induced (BCI) genes were upregulated in an NPRI-independent manner, whereas the expression levels of several pathogenesis-related $(P R)$ genes were elevated upon BTH treatment only in wNPRIOE. Two barley WRKY transcription factors, HvWRKY6 and $H v W R K Y 70$, were predicted and further validated as key regulators shared by the $\mathrm{BTH}$-induced resistance and the NPR1-mediated acquired resistance. Wheat transgenic lines overexpressing $\mathrm{HvWRKY6}$ and $\mathrm{HvWRKY70}$ showed different degrees of enhanced resistance to Puccinia striiformis f. sp.

$\mathrm{H}$. Li and J. Wu contributed equally to this work.

${ }^{\dagger}$ Corresponding authors: Xiaodong Wang; zhbwxd@ hebau.edu.cn; and Xiaojie Wang; wangxiaojie@ nwsuaf.edu.cn

Funding: This work was supported by the National Natural Science Foundation of China (31701776 and 31772150), the Provincial Natural Science Foundation of Hebei for Excellent Young Scholar (C2018204091), the China Agriculture Research System (CARS-3), the Open Project Program of State Key Laboratory of Crop Stress Biology for Arid Areas (CSBAAKF2018009), Shaanxi Innovation Team Project (2018TD-004), and the Supporting Program of Hebei Agricultural University for Oversea Talents (ZD201603).

*The $e$-Xtra logo stands for "electronic extra" and indicates that supplementary figures and supplementary tables are published online.

The author(s) declare no conflict of interest.

(C) (1) (3) Copyright $(2020$ The Author(s). This is an open access article distributed under the CC BY-NC-ND 4.0 International license. tritici pathotype CYR32 and Blumeria graminis f. sp. tritici pathotype E20. In conclusion, the transcriptional changes of BTH-induced resistance in barley were initially profiled, and the identified key regulators would be valuable resources for the genetic improvement of broad-spectrum disease resistance in wheat.

Keywords: barley, broad-spectrum disease resistance, BTH-induced resistance, defense signaling pathways, pathogenesis-related proteins, plant antifungal responses, salicylic acid, systemic acquired resistance, wheat

Systemic acquired resistance (SAR) provides broadspectrum disease resistance to secondary pathogenic invasions beyond the initial infection site. External treatments with salicylic acid (SA) or its chemical analog benzothiadiazole (BTH) can also stimulate SAR (Fu and Dong 2013). In Arabidopsis, the nonexpresser of pathogenesis-related genes 1 $(N P R 1)$ is reported as the key regulator of SAR, during which it associates with the TGACG-binding factor (TGA) transcription factors to induce the expression of downstream pathogenesisrelated (PR) genes (Dong 2004).

WRKY transcription factors played crucial roles in SAR in either NPR1-dependent or -independent ways. Using a microarray approach, AtWRKY18, AtWRKY58, and AtWRKY70 were identified as key regulatory nodes of SAR pathways in Arabidopsis (Wang et al. 2006). The expression level of the rice homolog of NPR1 ( $r N H 1)$ was regulated by OsWRKY3 and OsWRKY71 (Liu et al. 2005; Liu et al. 2007), whereas OsWRKY45 regulated the BTH-induced resistance in an $\mathrm{rNH1}$ independent pathway (Shimono et al. 2007). The transcriptional regulatory network of OsWRKY45 during the BTH-induced resistance has been further profiled using RNA sequencing (RNA-seq) analysis (Nakayama et al. 2013).

In one of our previous investigations, wheat homolog of NPR1 (wNPR1) showed much conserved protein interactions with homologs of TGA transcription factors from both wheat and rice (Cantu et al. 2013), which are essential for the functioning of NPR1 in other plant species (Chern et al. 2001; Després et al. 2003). Although the protein interactome of 
wNPR1 seems to be conserved, SAR in wheat and barley exhibited several unique features (Wang et al. 2018).

Systemic immunity, a SAR-like response in barley to secondary infection of Xanthomonas translucens pv. cerealis, could be induced in systemic uninfected leaves by infection of $X$. translucens pv. cerealis or Pseudomonas syringae pv. japonica in the elder leaves (Dey et al. 2014). However, the systemic immunity was not associated with local or systemic accumulation of SA but with a moderate local induction of jasmonate acid (JA) and, especially, abscisic acid (ABA). By applying RNA-seq analysis on the barley transgenic line knocking down HvNPRl (HvNPRl$K d$ ), the systemic immunity seemed to be regulated by several WRKY and ERF transcription factors in an NPRIindependent manner.

In the region adjacent to the infiltration area of $P$. syringae pv. tomato DC3000 in barley leaf, another form of SAR-like response to the secondary fungal pathogen Magnaporthe oryzae could be induced (Colebrook et al. 2012). The designated acquired resistance shared several common features with SAR in Arabidopsis, including induction of $P R$ genes and elevation of SA level. In our previous investigation, we observed significant associations between the inductions of $P R$ genes and the expression level of NPR1 during the P. syringae DC3000triggered acquired resistance (Wang et al. 2016). The results of our recent study showed that the acquired resistance to $M$. oryzae in barley was directly controlled by NPRl (Gao et al. 2018). In the same investigation, the transcriptome analysis results revealed the downstream genes of $N P R 1$ and key WRKY transcription factors during the acquired resistance response.

In several earlier studies, BTH treatment of Triticeae crops of wheat and barley induced broad-spectrum disease resistance to multiple fungal diseases, including leaf rust and powdery mildew (Beßer et al. 2000; Görlach et al. 1996; Hafez et al. 2014). Different from BTH-induced SAR in Arabidopsis, few $P R$ genes were sensitive to BTH treatment, whereas the expression levels of another group of genes, including wheat chemicalinduced $(W C I)$ and barley chemical-induced $(B C I)$ genes, were highly expressed (Beßer et al. 2000; Molina et al. 1999; Vallélian-Bindschedler et al. 1998). Interestingly, a faster activation of disease resistance to Fusarium head blight was observed in a wheat transgenic line overexpressing Arabidopsis AtNPRl, and the expression of TaPRl became more sensitive to the BTH treatment (Makandar et al. 2006). Nevertheless, the regulatory network of the BTH-induced resistance, as well as the specific role of NPR1 during such SAR-like responses, was largely unknown.

In the present investigation, transcriptome sequencing was performed on barley transgenic lines overexpressing wheat wNPRl ( $w N P R 1-O E)$ and HvNPRl-Kd to reveal the role of $N P R l$ during the BTH-induced resistance. Most of the $B C I$ genes were regulated independently from $N P R 1$, whereas few $P R$ genes became more sensitive to BTH only in $w N P R 1-O E$. Two differentially expressed $W R K Y$ transcription factors, $H v W R K Y 6$ and $H v W R K Y 70$, were selected for further functional characterization and both of them showed great potential in the improvement of broad-spectrum disease resistance in wheat.

\section{RESULTS}

Transcriptome analysis of $w N P R 1-O E$ and $H v N P R 1-K d$ during the BTH-induced resistance.

The wheat and barley NPR1 homologs seemed to be very conserved with previously reported rice NPR1 protein (rNH1, GenBank accession AAX18700.1) (Supplementary Fig. S1). The similarities between barley HvNPR1 and wheat NPR1 homologs deduced from A, B, and D subgenome copies were $92.72,91.68$, and $91.03 \%$, respectively, indicating a very conserved role of NPR1 in these relative plant species. To explore the role of NPR1 during BTH-induced resistance in barley, we applied transcriptome analysis on samples harvested from BTH-sprayed third leaves of barley transgenic lines wNPRl$O E-E 1-T 3$ (wheat $w N P R 1$ overexpressing line, independent transgenic event number $1, \mathrm{~T} 3$ generation) and $H v N P R 1-K d$ E1-T6 (barley HvNPR1 knockdown line, independent transgenic event number 1, T6 generation), as well as the wild-type plants. Samples from the water-sprayed wild-type plants served as a control. The transcript abundance of the NPRI transgene was determined by quantitative reverse-transcription (qRT)PCR assay. The barley elongation factor 1a (HvEF la, GenBank accession Z50789) was used as an internal reference gene. Compared with the wild-type plants, approximately 4.3 -fold more and 0.4-fold less NPR1 transcripts were detected in the wNPR1-OE-E1-T3 and HvNPR1-Kd-E1-T6, respectively (Supplementary Fig. S2). Each of the materials included four biological replicates and, in total, 16 samples were subjected to a 12-Gb Illumina sequencing (Supplementary Table S1). For each of the samples, more than 58 million 150-bp pair-end reads were collected. The genome of Hordeum vulgare from Ensembl Genomes was utilized for the assembly of the transcriptome. Briefly, in total, 32,247 genes were mapped on the genome sequence (Supplementary Table S2). We observed significant $\left(R^{2}>0.92\right)$ correlations of the overall gene expression levels between biological replicates (Supplementary Fig. S3). The fragments per kilobase of transcript per million mapped reads (FPKM) value was employed to estimate the abundance of transcripts. Raw reads for the transcriptome were stored at NCBI BioProject PRJNA509975.

\section{The expression patterns of $P R$ and $B C I$ genes} during the BTH-induced resistance in barley.

The expression patterns of all $18 P R$ gene families were initially profiled by searching their FPKM values in our transcriptome database (Fig. 1). We found that BTH treatment was sufficient to elevate the expression levels of $H v P R 1, H v P R 2$, HvPR3 (Chit $2 a$ and Chit2b), HvPR5 (TLP6, TLP7, and TLP8), $H v P R 14$, and $H v P R 17 b$. The sensitivities of all such genes to BTH treatment were dramatically increased in wNPR1-OE. On the other hand, most of the $B C I$ genes were highly induced upon BTH treatment in an NPRI-independent manner (Fig. 1).

We then performed a qRT-PCR assay to validate the gene expression patterns of $P R$ and $B C I$ genes determined by FPKM values. Two independent lines for each of the transgenic materials were included. The transcriptional abundances of NPRI transgene, $H v P R 1, H v P R 2, H v B C I 1, H v B C I 3$, and $H v B C I 7$ were monitored and expressed as linearized fold $H v E F 1 a$ levels (Fig. 2; Supplementary Fig. S2). The expression levels of $H v B C I 1$ (**P $=0.0021), H v B C I 3$ (**P $=0.0008$ ), and $H v B C I 7$ (** $P=0.0054)$ were significantly induced by the BTH treatment in an NPRI-independent manner. Of note, the expression levels of HvPRI (*** P<0.0001) and HvPR2 (*** $P<0.0001)$ were significantly upregulated only in $w N P R 1-O E$ upon the BTH treatment.

\section{Regulatory network of NPRI during the BTH-induced resistance.}

DESeq2 was applied to identify significant upregulated ( $q$ value $<0.05$ and $\log _{2}$-fold change $>1$ ) differentially expressed genes (DEGs) in the comparisons of WT_BTH versus WT_CK, OE_BTH versus WT_BTH, and Kd_BTH versus WT_BTH. In total, 847 genes in the wild-type plants were significantly upregulated upon the BTH treatment. Among these DEGs, the expression levels of 104 genes were much more highly elevated 
in the $w N P R 1-O E$ than those in the wild-type plants, which were designated as type I DEGs (Supplementary Fig. S4; Supplementary Table S3). We speculated that the type I DEGs were downstream genes of the NPRl-mediated signaling pathway, triggered by the BTH treatment. DEGs encoding WRKY transcription factors, glucan endo-1,3- $\beta$-glucosidase, and various protein kinases were enriched in this group. Gene ontology (GO) analysis showed that the type I DEGs were annotated with "catalytic activity" and "binding" in molecular function category and with "metabolic process" in biological process category (Supplementary Fig. S5A). The remaining 743 upregulated genes were predicted to be NPRI-independent BTH-sensitive genes, and designated as type II DEGs (Supplementary Figs. S4 and S5B; Supplementary Table S4). Interestingly, we also detected, in total, 107 genes more highly expressed in the $H v N P R l-K d$ than those in the wild-type plants upon BTH treatment. Among these DEGs, 73 genes seemed to be uniquely induced in $H v N P R I-K d$, which were designated as type III DEGs (Supplementary Figs. S4 and S5C; Supplementary Table S5).

\section{HvWRKY6 and HvWRKY70 were predicted as key transcriptional regulators shared by BTH-induced resistance and the NPRI-mediated acquired resistance.}

In the present transcriptome database, 46 WRKY genes were identified, roughly half of which were significantly induced upon BTH treatment (Fig. 3). All of the identified WRKY genes were temporarily designated based on their closest homologs in relative plant species. Most of the induced WRKY genes seemed to be regulated in an NPRl-independent manner. However, among the differentially expressed $W R K Y$ genes, the induction levels of two genes, MLOC_66134 and MLOC_78461, were higher in $w N P R 1-O E$ than those in the wild-type plants (Supplementary Fig. S6A). We then double-checked the expression profiles of these two genes in one of our previous transcriptome databases referring to $P$. syringae DC3000-triggered acquired resistance (Gao et al. 2018). Based on their FPKM values, we found that $H v W R K Y 6$ and $H v W R K Y 70$ were also more highly induced in $w N P R 1-O E$ than in the wild-type plants upon $P$. syringae DC3000 treatment (Supplementary Fig. S6B). Based on the results, we speculated that these two WRKY genes might serve as key transcriptional regulators shared by BTHinduced resistance and NPRl-mediated acquired resistance.

The closest homologs of the deduced proteins of MLOC_66134 and MLOC_78461 in other plant species, including Triticum aestivum (Ta), Hordeum vulgare (Hv), Aegilops tauschii (Att), T. uratu, Oryza sativa, and Arabidopsis thaliana (At), were collected from GenBank and wheat genome databases. Furthermore, a neighbor-joining tree was generated (Supplementary Fig. S7). We temporarily designated the $M L O C_{-}$ 78461 gene as the barley $H v W R K Y 6$ based on its closest homolog, AttWRKY6 in Aegilops tauschii, and MLOC_66134 as barley $H v W R K Y 70$, referring to AttWRKY70 in A. tauschii. The closest homolog of HvWRKY6 protein in a common wheat genome database (TRIAE_CS42_2DS_TGACv1_179236_AA0605510) was clustered with another protein, AttWRKY72 from A. tauschii, and the closest homolog of HvWRKY70 in common wheat (TRIAE_CS42_7DL_TGACv1_602597_AA1962010) was clustered with a recently identified TaWRKY146 protein (Ma et al. 2017).

To investigate the subcellular localization of HvWRKY6 and HvWRKY70 proteins, a green fluorescent protein (GFP) tag was fused to each end of the HvWRKY6 and HvWRKY70 proteins, and the recombinant transcripts were transiently expressed in Nicotiana benthamiana using Agrobacterium. Clear fluorescence for HvWRKY6-GFP, GFP-HvWRKY6, and GFP-HvWRKY70 was observed in the nucleus (Supplementary
Fig. S8). On the other hand, for HvWRKY70-GFP, GFP signals were detected in both the nucleus and the cytoplasm, indicating a block of nuclear localization signal of HvWRKY70 by GFP tag at the C-terminal end.

\section{Wheat transgenic line overexpressing the HvWRKY6 gene exhibited promoted resistance to $M$. oryzae in a $P R$ gene-associated manner.}

To explore the potential application of the HvWRKY6 and $H v W R K Y 70$ genes, we generated wheat transgenic lines overexpressing the $H v W R K Y 6$ or $H v W R K Y 70$ gene under the maize Ubiquitin promoter (HvWRKY6-OE and HvWRKY70-OE) in the genetic background of spring common wheat JW1. The expression levels of the HvWRKY6 transgene were from 2.3- to 7.2-fold of the levels of the TaActin endogenous control (Supplementary Fig. S9A), and those of $H v W R K Y 70$ transgene were from 0.4- to 1.4-fold (Supplementary Fig. S9B). We did not detect any signals of $H v W R K Y 6$ and $H v W R K Y 70$ in the wildtype plants.

We then utilized the $M$. oryzae isolate P131 to investigate the degree of acquired resistance induced by $P$. syringae DC3000 in $H v W R K Y 6-O E$ and $H v W R K Y 70-O E$ (Fig. 4A). The third leaves of wheat seedlings were infiltrated with either $P$. syringae DC3000 or sterile water as a control. M. oryzae isolate P131 was inoculated as the secondary pathogen in the region adjacent to the $P$. syringae DC3000 infiltration area at 2 days postinfiltration (dpi). In the wild-type plants, acquired resistance triggered by $P$. syringae DC3000 significantly $(* * P<0.01)$

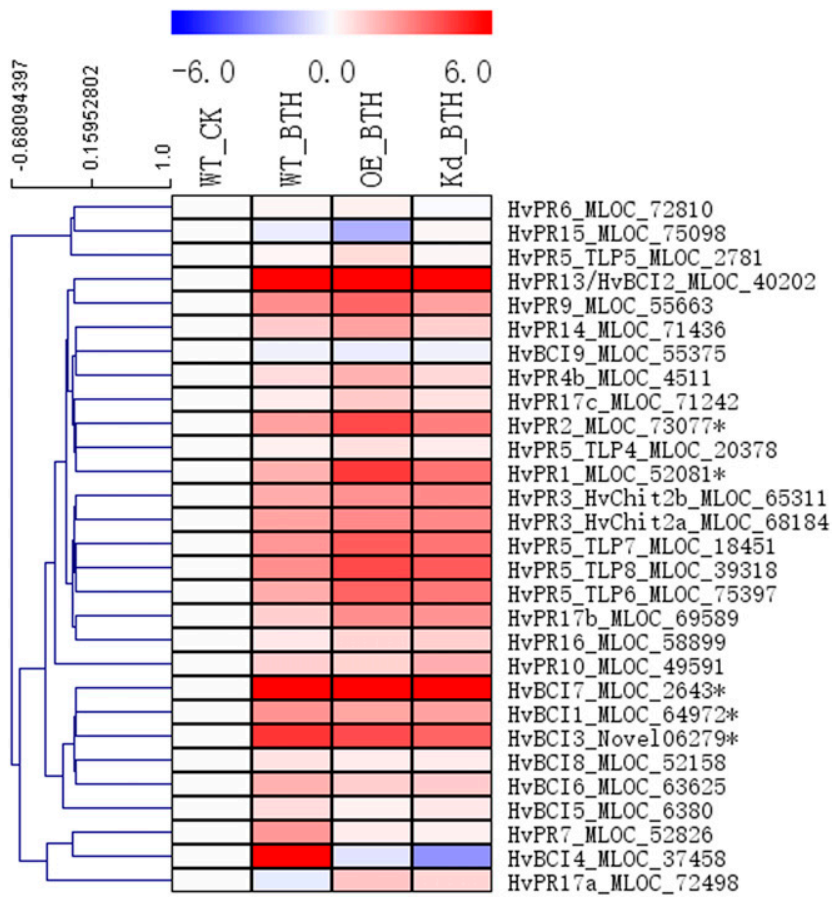

Fig. 1. Expression patterns of pathogenesis-related $(P R)$ and barley chemical-induced $(B C I)$ genes during benzothiadiazole (BTH)-induced resistance were initially profiled. Fragments per kilobase of transcript per million mapped reads values for each of the transcripts in WT_BTH, $\mathrm{OE}$ BTH, and Kd_BTH were relative to that in WT_CK. Data were transformed into $\log _{2}$-fold change format using Microsoft Excel software. A heatmap was created using $\mathrm{MeV}$ software. The function "Hierarchical Clustering" in MeV software was employed to cluster genes with similar expression patterns. Five genes were selected (labeled with an asterisk [*]) for further quantitative reverse-transcription $\mathrm{PCR}$ validation assay. $\mathrm{OE}=$ overexpressing wheat $w N P R 1$ ( $w N P R 1-O E), \mathrm{Kd}=$ knocking down barley HvNPR1 $(H v N P R 1-K d)$, WT = wild type, CK = mock-inoculation with water, and NPR1 = nonexpresser of pathogenesis-related genes 1 . 
decreased the lesion size of M. oryzae isolate P131 (Fig. 4A). Compared with the wild-type plants, we observed more pronounced $(* P<0.05)$ resistance to $M$. oryzae in HvWRKY6-OE, even in the mock control (Fig. 4A; Supplementary Table S6), which indicated that HvWRKY6 improved the basal resistance of wheat. On the other hand, we did not observe any elevated resistance to $M$. oryzae during the acquired resistance in the wheat transgenic line $H v W R K Y 70-O E$ (Fig. 4A).

To investigate the possible regulatory downstream genes of $H v W R K Y 6$ during the NPRl-mediated acquired resistance, a series of qRT-PCR assays was performed. P. syringae DC3000 was applied to induce the acquired resistance in $H v W R K Y 6-O E$ and $H v W R K Y 70-O E$, as well as the wild-type plants. Water infiltration served as a mock control. RNA samples were harvested from the region adjacent to the $P$. syringae DC3000 infiltration area at $2 \mathrm{dpi}$. The expression levels of TaPRla, $T a P R 2$, and $T a P R 4 b$, were determined by qRT-PCR assay. The wheat TaActin gene served as a reference gene. Significant inductions of TaPRla $(* * P=0.0001), \operatorname{TaPR} 2(* P=0.0106)$, and $T a P R 4 b(* * P=0.0066)$, were detected during the acquired resistance in the wild-type plants (Fig. 4B; Supplementary Fig. $\mathrm{S} 10)$. Interestingly, we found that the expression levels of such $P R$ genes in the mock control of the wheat transgenic line $H v W R K Y 6-O E$ were significantly $(* P<0.05)$ higher than that in the wild-type plants (Fig. 4B). The whole experiment was repeated twice with two independent lines of HvWRKY6-OE,
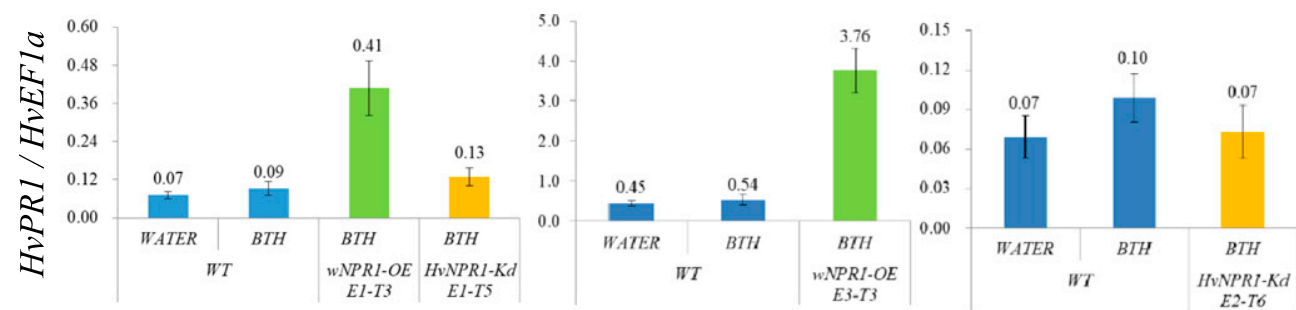

Combined $P$ value:

WT: $\mathrm{BTH}$ vs WATER $=0.2071$

BTH: $w N P R 1-O E$ vs WT $<\mathbf{0 . 0 0 0 1} * * *$

BTH: $H v N P R 1-K d$ vs $\mathrm{WT}=0.6838$
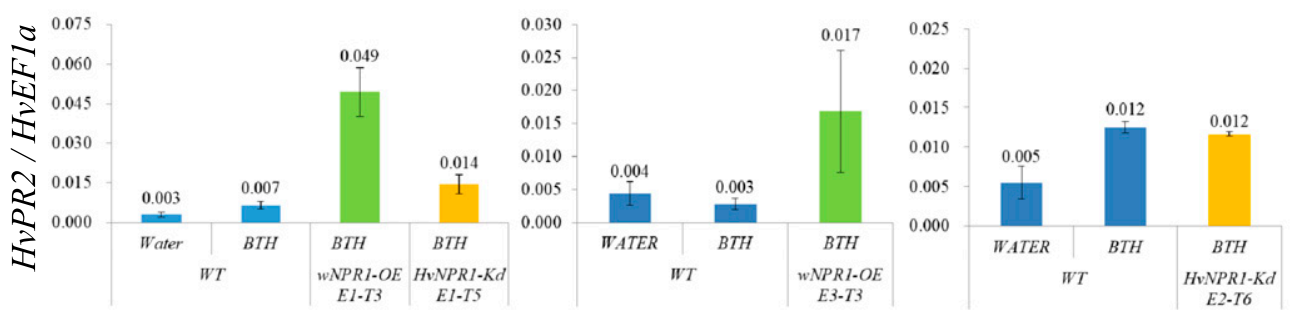

Combined $P$ value:

WT: $\mathrm{BTH}$ vs WATER $=0.0517$

BTH: $w N P R I-O E$ vs WT $<\mathbf{0 . 0 0 0 1 * * *}$

BTH: $H v N P R 1-K d$ vs WT $=0.1116$
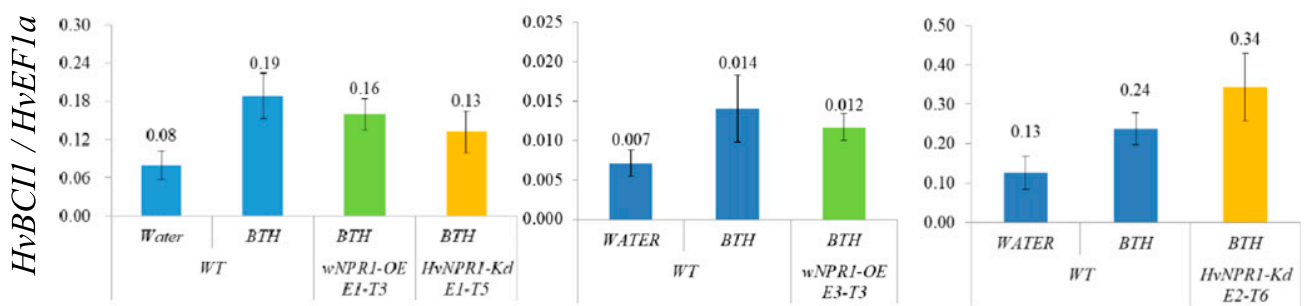

Combined $P$ value:

WT: $\mathrm{BTH}$ vs WATER $=\mathbf{0 . 0 0 2 1}$ **

BTH: $w N P R 1-O E$ vs $\mathrm{WT}=0.5904$

BTH: $H v N P R I-K d$ vs WT $=0.9951$
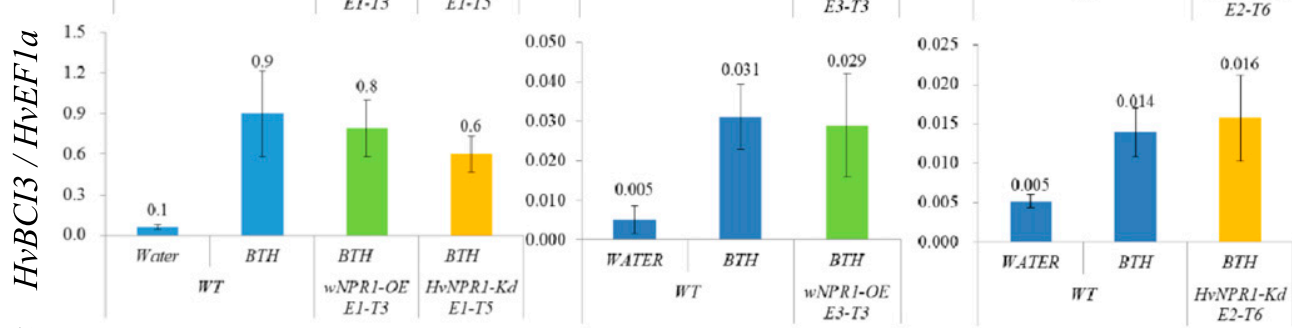

Combined $P$ value:

WT: $\mathrm{BTH}$ vs WATER $=\mathbf{0 . 0 0 0 8}$ **

BTH: $w N P R 1-O E$ vs $\mathrm{WT}=0.0812$

BTH: $H v N P R I-K d$ vs WT $=0.8520$
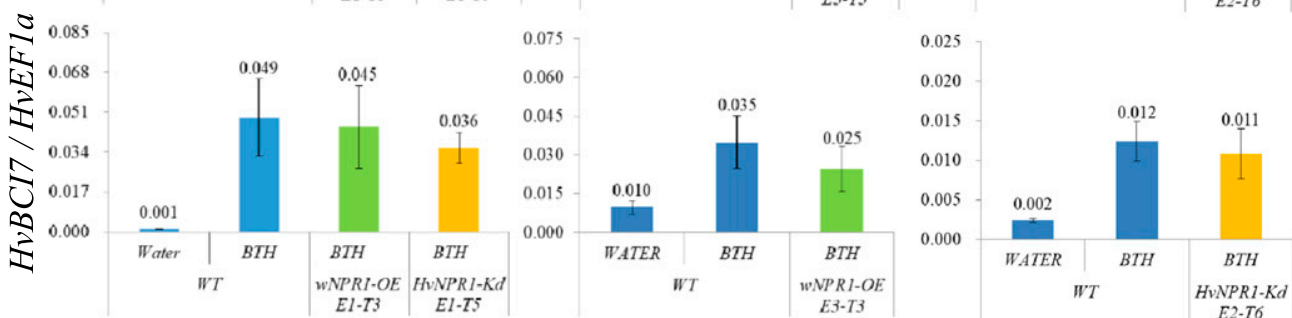

Combined $P$ value:

WT: $\mathrm{BTH}$ vs WATER $=\mathbf{0 . 0 0 5 4}$ *

BTH: $w N P R 1-O E$ vs WT $=0.5977$

BTH: HvNPRl-Kd vs WT $=0.5417$

Fig. 2. Expression levels of selected pathogenesis-related $(P R)$ and barley chemical-induced $(B C I)$ genes in barley transgenic lines overexpressing wheat wNPRI (wNPR1-OE) and knocking down barley HvNPRI (HvNPR1-Kd) during the benzothiadiazole (BTH)-induced resistance were validated by a quantitative reverse-transcription PCR assay. Third leaves of $w N P R 1-O E$ and $H v N P R 1-K d$, as well as wild-type (WT) plants, were sprayed with $0.1 \mathrm{mM} \mathrm{BTH}$ Water-sprayed WT plants served as a control. RNA samples were harvested at $48 \mathrm{~h}$ posttreatment. The whole experiment was systemically repeated three times using independent transgenic lines (left, middle, and right panels) and each transgenic line consisted of six to 10 biological replicates. The relative transcript abundance was expressed relative to that of the internal reference $H v E F l a$ following the $2^{-\Delta \mathrm{Ct}}$ method. Mean and standard error for the relative expression levels were calculated using Microsoft Excel software. A general linearized model analysis of variance $(*, * *$, and $* * *$ indicate $P<0.05,0.01$, and 0.0001 , respectively) was performed using SAS v9.4 software. OE = overexpressing transgenic line, $\mathrm{Kd}=$ knockdown transgenic line, $\mathrm{E}=$ independent transgenic event, and $\mathrm{T}=$ generation of the transgenic material. 
and each repeat included six to eight biological replicates. On the other hand, we did not observe any significant differences in the expression levels of the selected $P R$ genes between the wheat transgenic line $H v W R K Y 70-O E$ and the wild-type plants (Supplementary Fig. S10).

$P R$ and $B C I$ genes in wheat transgenic line $H v W R K Y 70-O E$ became more sensitive to the BTH treatment.

To investigate the roles of $H v W R K Y 6$ and $H v W R K Y 70$ genes during the BTH-induced resistance, we further performed qRTPCR assays on HvWRKY6-OE and HvWRKY70-OE. Water treatment served as a mock control. Two days posttreatment (dpt) with $\mathrm{BTH}$, the expression levels of TaPRla, TaPR2, $T a P R 4 b, T a B C I 1$, and TaBCI3 were monitored by qRT-PCR assay (Fig. 5). In the wild-type plants, only the expression levels of TaBCII (***P $<0.0001)$ and TaBCI3 $(* * * P<0.0001)$ genes were significantly induced upon the BTH treatment. However, the expression levels of all of the tested $P R$ and $B C I$ genes were significantly $(* * P<0.01)$ higher in the $H v W R K Y 70-O E$ than those in the wild-type plants. The whole experiment was systemically repeated twice with two independent lines of $H v W R K Y 70-O E$, and each line included five to seven biological replicates. On the other hand, we did not observe any significant differences in the expression levels of the selected $P R$ and $B C I$ genes between the HvWRKY6-OE and the wild-type plants after the BTH treatment (Fig. 5).

The genomic regions of TaPRla, TaPR2, TaPR $4 b, T a B C I 1$, and $T a B C I 3$ genes were collected from the wheat genome database (Chinese Spring, TGACv1 version) and were subjected to analysis of the transcription factor-binding site in their promoter regions (Supplementary Table S7). We did not detect any WRKY transcription factor-binding sites in the tested $P R$ and $B C I$ genes, which indicated that HvWRKY6 and HvWRKY70 might regulate these genes indirectly.

\section{HvWRKYG and HvWRKY70 improved}

wheat resistance to stripe rust and powdery mildew.

We hypothesized that the identified HvWRKY6 and $H v W R K Y 70$ might improve broad-spectrum disease resistance in wheat. The generated wheat transgenic lines were then subjected to inoculation with both wheat stripe rust (Puccinia striiformis f. sp. tritici) and powdery mildew (Blumeria graminis f. sp. tritici), both of which cause severe fungal diseases threatening global wheat production. For the stripe rust inoculation, the wheat seedlings of the wild-type JW1 plants showed phenotypes highly susceptible (full sporulation) to the virulent $P$. striiformis f. sp. tritici pathotype CYR32 at $15 \mathrm{dpi}$ (Fig. 6). The proportion of $P$. striiformis f. sp. tritici sporulation area on each of the collected leaves was recorded using ASSESS software. The sporulation of the rust fungi was significantly $(* P<0.05$ and $* * P<0.01)$ diminished or delayed in the wheat transgenic lines HvWRKY6-OE and HvWRKY70-OE compared with the wild-type plants (Fig. 6). On the other hand, for the powdery mildew inoculation, the wild-type plants exhibited phenotypes highly susceptible (full sporulation) to the prevalent $B$. graminis f. sp. tritici isolate E20 at 15 dpi (Fig. 7). Compared with the wild-type plants, the proportion of $B$. graminis f. sp. tritici sporulation area on each of the collected leaves was significantly $(* P<0.05$ and $* * P<0.01)$ lower in the wheat transgenic line $H v W R K Y 70-O E$ (Fig. 7). We did not observe any enhanced resistance to $B$. graminis f. sp. tritici in the wheat transgenic line HvWRKY6-OE.

\section{DISCUSSION}

SAR triggered by initial pathogen infection or BTH treatment is considered to confer plant broad-spectrum resistance to secondary pathogenic invasions (Klessig et al. 2018). However, due to the large genome size and the complex gene homolog network, to date, SAR in Triticeae crops (wheat and barley) has been poorly explored (Wang et al. 2018). The acquired resistance induced by Pseudomonas syringae DC3000 shared several key features with the SAR described in Arabidopsis (e.g., inductions of $P R$ genes, elevation of endogenous SA, and involvement of NPRI) (Colebrook et al. 2012; Gao et al. 2018). Meanwhile, BTH-induced resistance was sufficient to improve broad-spectrum disease resistance in wheat and barley to multiple pathogens in a $P R$ gene-independent manner (Beßer et al. 2000; Görlach et al. 1996; Hafez et al. 2014; Molina et al. 1999; Vallélian-Bindschedler et al. 1998). The regulatory

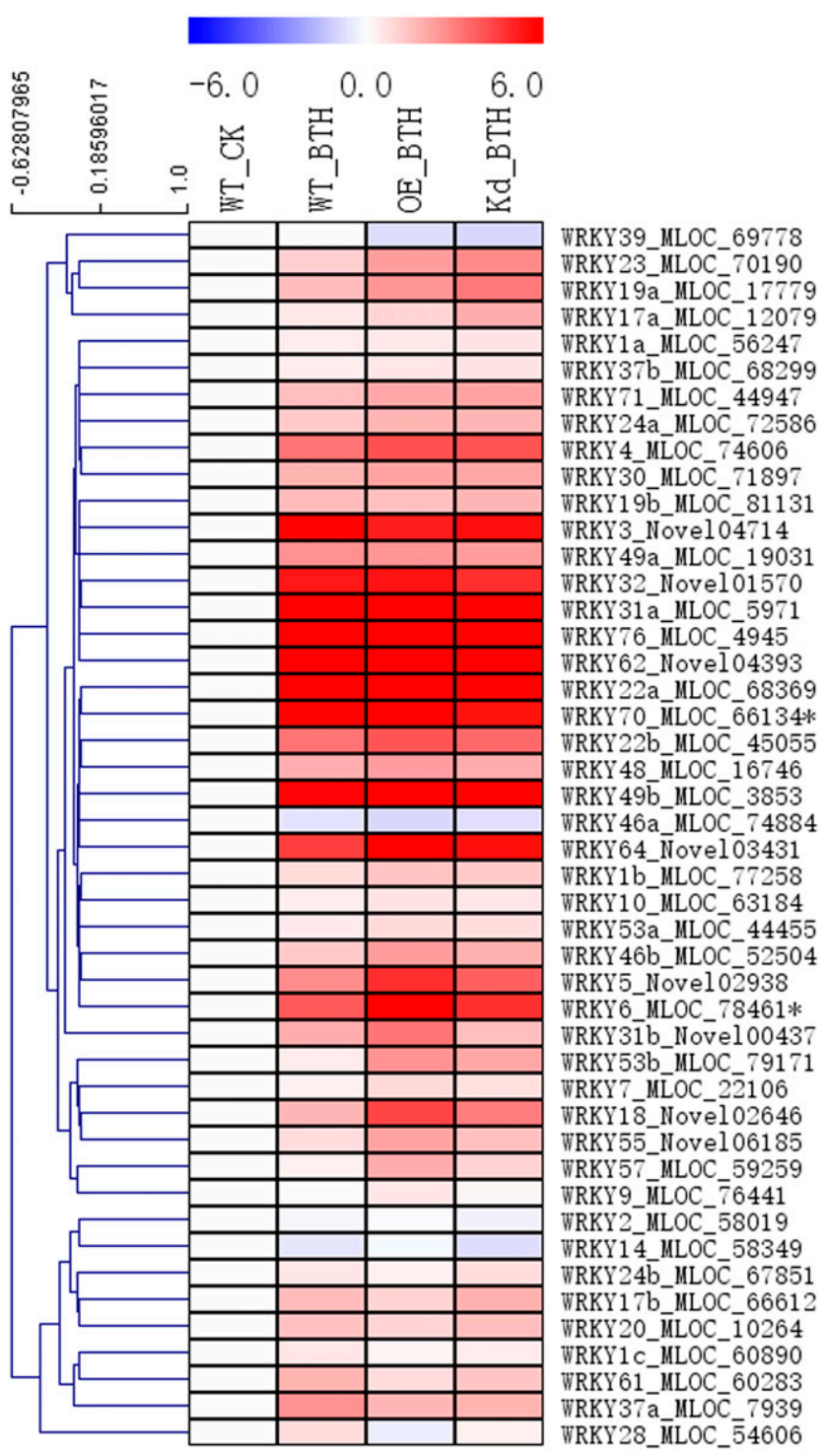

Fig. 3. Genome-wide expression profiles of WRKY genes during benzothiadiazole (BTH)-induced resistance. Fragments per kilobase of transcript per million mapped reads values for each of the transcripts in WT_BTH, OE_BTH, and Kd_BTH were relative to that in WT_CK. Data were transformed into $\log _{2}$-fold change format using Microsoft Excel software. A heatmap was created using $\mathrm{MeV}$ software. The function "Hierarchical Clustering" in MeV software was employed to cluster genes with similar expression patterns. Names of the $W R K Y$ genes were temporally designated based on their closest homologs in relative plant species. HvWRKY6 and $H v W R K Y 70$ were chosen (labeled with an asterisk [*]) for functional exploration. $\mathrm{OE}=$ overexpressing wheat $w N P R 1$ ( $w N P R 1-O E), \mathrm{Kd}=$ knocking down barley $H v N P R 1(H v N P R 1-K d)$, and CK = mock inoculation with water. 
networks of BTH-induced resistance in wheat and barley were largely unknown.

To investigate the role of NPRI during BTH-induced resistance, we established a transcriptome database by sequencing RNA samples collected from BTH-sprayed barely transgenic lines ( $w N P R 1-O E$ and $H v N P R l-K d$ ) and wild-type plants. Based on the expression profiles generated from the transcriptome database and subsequent qRT-PCR validation (Figs. 1 and 2), most of the $B C I$ genes were highly expressed independently from NPR1. It is noteworthy that $P R$ genes became

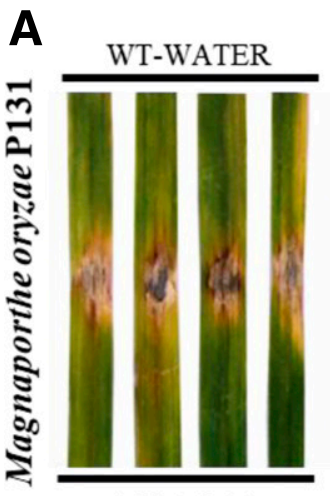

$0.73 \pm 0.04$

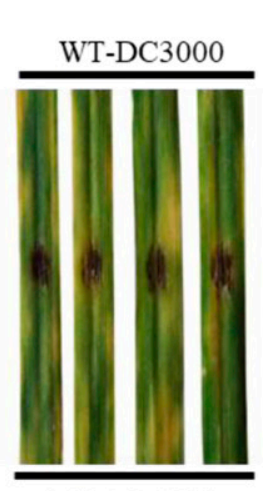

$0.42 \pm 0.02 * *$

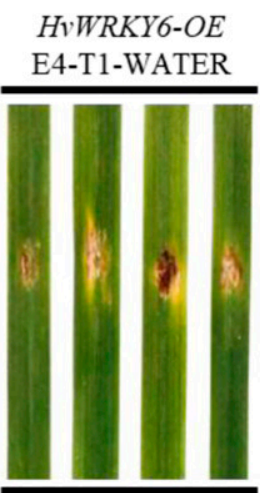

$0.52 \pm 0.05 * *$
HvWRKY6-OE
E4-T1-DC3000

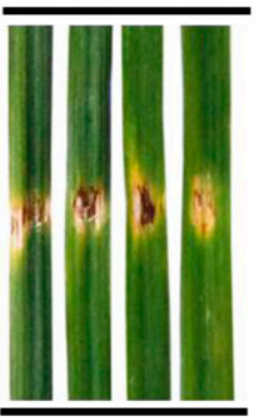

$0.55 \pm 0.04 * *$
HvWRKY70-OE E2-T1-WATER

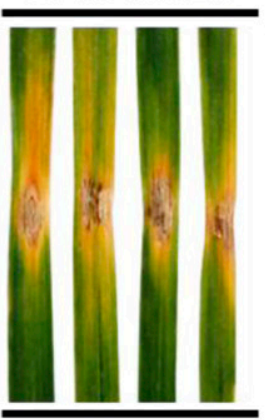

$0.80 \pm 0.09$
HvWRKY70-OE E2-T1-DC3000

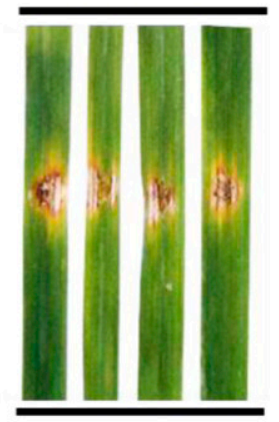

$0.60 \pm 0.03 *$

B
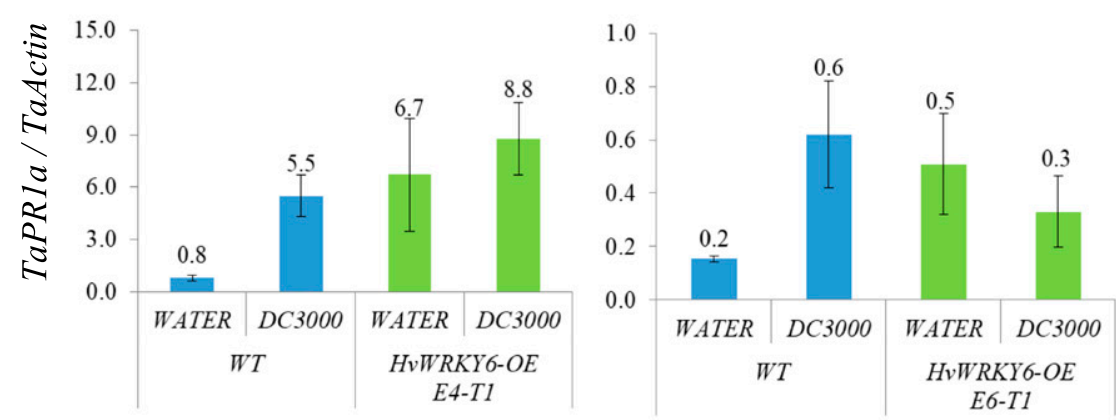

Combined $P$ value:

WT: DC3000 vs WATER $=\mathbf{0 . 0 0 0 1} *$ *

WATER: $H v W R K Y 6-O E$ vs $\mathrm{WT}=\mathbf{0 . 0 2 1 9}$ *

DC3000: $H v W R K Y 6-O E$ vs WT $=0.7082$
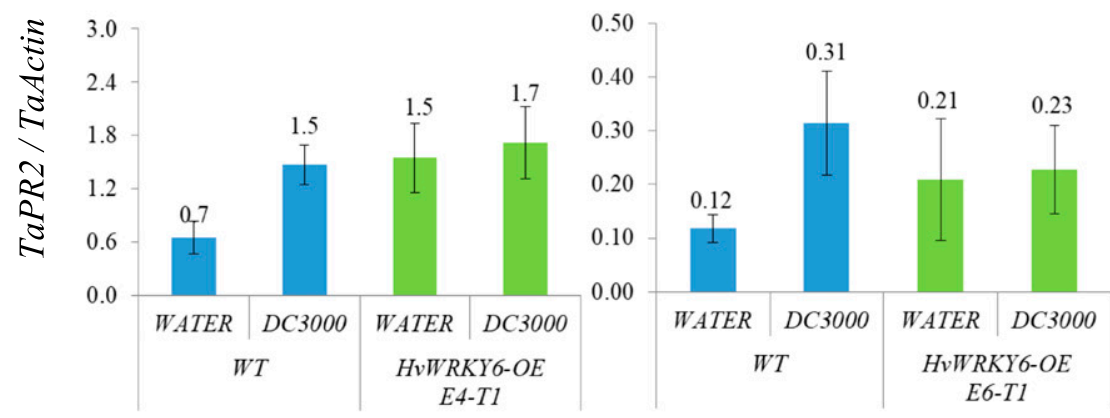

Combined $P$ value:

WT: DC3000 vs WATER $=\mathbf{0 . 0 1 0 6}$ *

WATER: $H \nu W R K Y 6-O E$ vs WT $=\mathbf{0 . 0 4 5 6 *}$ DC3000: $H v W R K Y 6-O E$ vs WT $=0.9702$
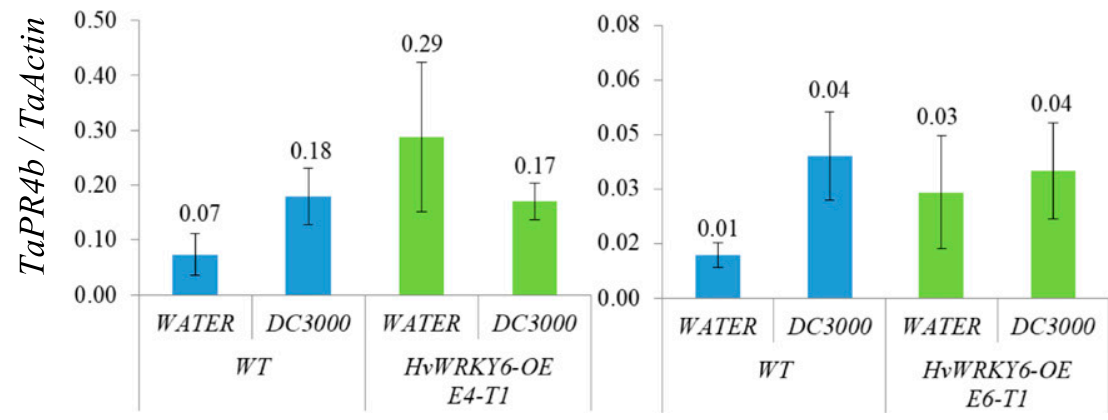

Combined $P$ value:

WT: DC3000 vs WATER $=\mathbf{0 . 0 0 6 6} * *$

WATER: $H v W R K Y 6-O E$ vs WT $=\mathbf{0 . 0 4 4 3} *$ DC3000: $H v W R K Y 6-O E$ vs WT $=0.6999$

Fig. 4. Wheat transgenic line overexpressing the HvWRKY6 gene showed promoted resistance to Magnaporthe oryzae in a pathogenesis-related (PR) geneassociated manner. Third leaves of wheat transgenic lines HvWRKY6-OE and $H v W R K Y 70-O E$, as well as wild-type (WT) plants, were infiltrated with either Pseudomonas syringae DC3000 or water as a control. A, M. oryzae isolate P131 was inoculated as the secondary pathogen in the region adjacent to the $P$. syringae DC3000 infiltration area at $48 \mathrm{~h}$ postinfiltration (hpi). Numbers represent the average lesion size of M. oryzae P131. The whole experiment was repeated twice with two independent lines of HvWRKY6-OE and each line included six to eight biological replicates (Supplementary Table S6). A Dunnett's test (* and ** indicate $P<0.05$ and 0.01 , respectively) was performed using SAS v9.4 software. B, Transcript levels of TaPR1, TaPR2, and TaPR4b in the wheat transgenic line HvWRKY6-OE during acquired resistance. RNA samples were harvested from the region adjacent to the $P$. syringae DC3000 infiltration area at $48 \mathrm{hpi}$. The experiment consisted of five to six biological replicates. The relative transcript abundance was expressed relative to that of the internal reference TaActin following the $2^{-\Delta \mathrm{Ct}}$ method. Mean and standard error for the relative expression levels were calculated using Microsoft Excel software. A general linearized model analysis of variance (* and $* *$ indicate $P<0.05$ and 0.01 , respectively) was performed using SAS v9.4 software. 
more sensitive to BTH treatment only in wNPR1-OE, which was consistent with previously reported higher expression of the TaPRI gene in a wheat transgenic line overexpressing AtNPRl after the BTH treatment (Makandar et al. 2006). A large number of DEGs were identified in our subsequent analysis; especially, most of the BTH-sensitive genes (type II DEGs) seemed to have been induced in an NPRl-independent manner (Supplementary Fig. S4; Supplementary Table S4). Based on this evidence, we
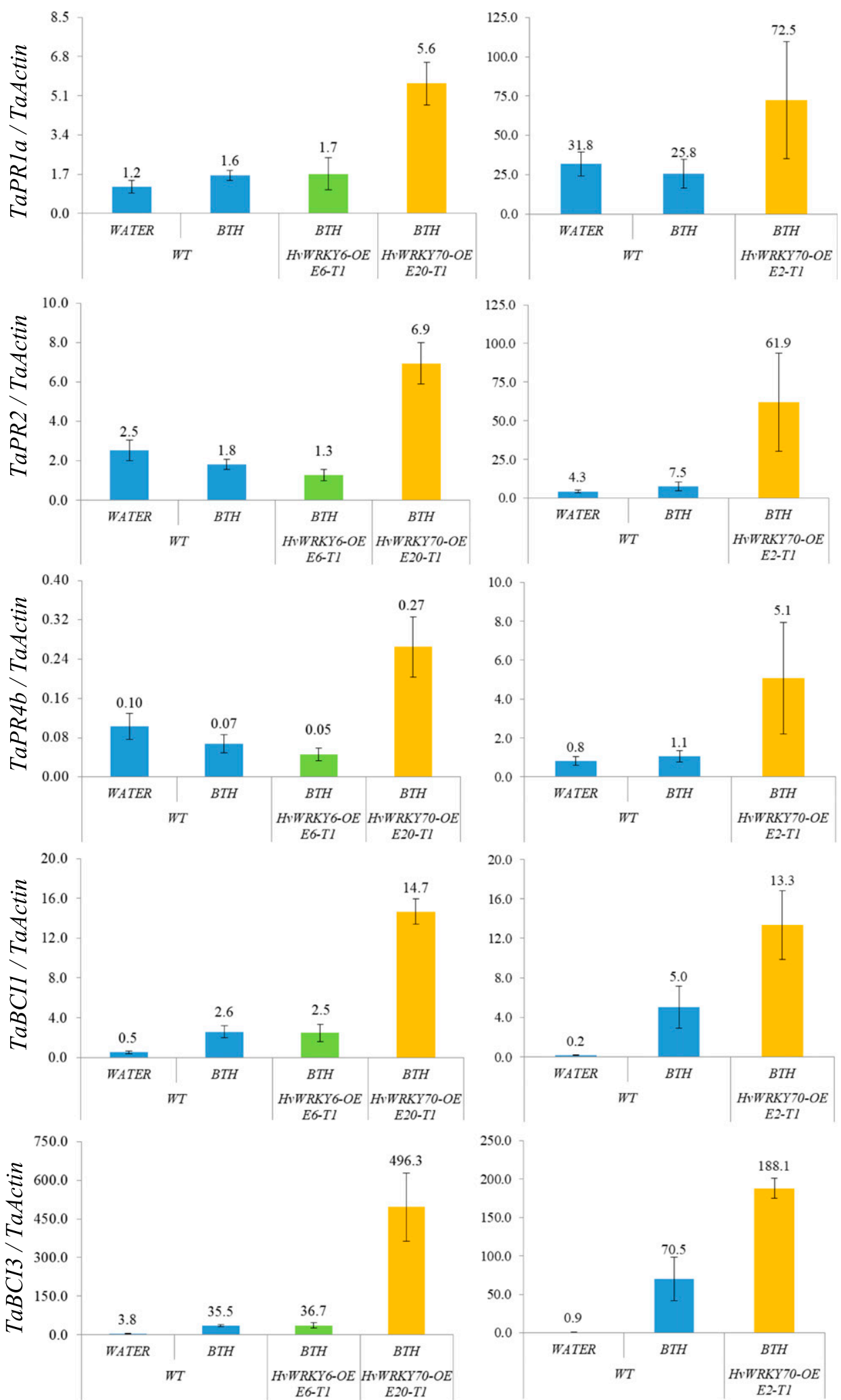

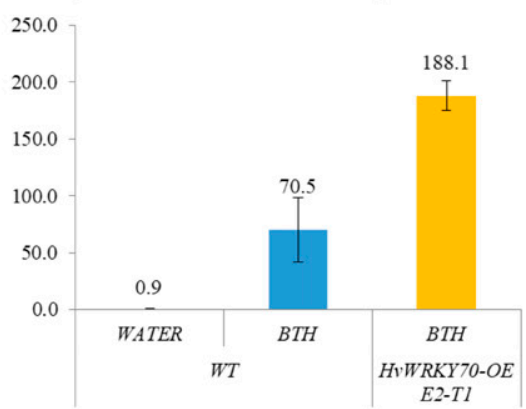

Combined $P$ value: WT: $\mathrm{BTH}$ vs WATER $=0.8667$ BTH: $H v W R K Y 6-O E$ vs WT $=0.9425$ BTH: HvWRKY70-OE vs WT $=\mathbf{0 . 0 0 3 9} * *$

Combined $P$ value: WT: $\mathrm{BTH}$ vs WATER $=0.9036$ BTH: HvWRKY6-OE vs WT $=0.2037$ BTH: $H v W R K Y 70-O E$ vs WT $=\mathbf{0 . 0 0 0 2} * *$

Combined $P$ value: WT: $\mathrm{BTH}$ vs WATER $=0.7687$ BTH: $H v W R K Y 6-O E$ vs WT $=0.3663$ BTH: $H v W R K Y 70-O E$ vs WT $=\mathbf{0 . 0 0 0 4} * *$

Combined $P$ value: WT: BTH vs WATER $<\mathbf{0 . 0 0 0 1 * * *}$ BTH: $H v W R K Y 6-O E$ vs WT $=0.9357$ BTH: $H v W R K Y 70-O E$ vs $W T=\mathbf{0 . 0 0 0 4}$ **

Combined $P$ value: WT: BTH vs WATER $<\mathbf{0 . 0 0 0 1} * * *$ BTH: $H v W R K Y 6-O E$ vs WT $=0.9242$ BTH: HvWRKY70-OE vs WT $=\mathbf{0 . 0 0 0 6}$ **

Fig. 5. Pathogenesis-related $(P R)$ and barley chemical-induced $(B C I)$ genes became more sensitive to benzothiadiazole (BTH) treatment in $H v W R K Y 70-O E$. Third leaves of wheat transgenic lines HvWRKY6-OE and HvWRKY70-OE, as well as wild-type (WT) plants, were sprayed with 0.1 mM BTH. Samples from water-sprayed WT plants served as a control. RNA samples were harvested at $48 \mathrm{~h}$ posttreatment. The whole experiment was systemically repeated twice with two independent transgenic lines, and each line included five to seven biological replicates. The relative transcript abundance was expressed relative to that of the internal reference TaActin following the $2^{-\Delta \mathrm{Ct}}$ method. Mean and standard error for the relative expression levels were calculated using Microsoft Excel software. A general linearized model analysis of variance (* and ** indicate $P<0.05$ and 0.01 , respectively) was performed using SAS v9.4 software. 
speculated that NPRI and its regulated $P R$ genes were partially involved in the BTH-induced transcriptional response in wheat and barley. In Arabidopsis, roughly half of the BTH-sensitive genes were elevated in an NPRl-dependent manner, some of which were further regulated by WRKY18 transcription factor (Wang et al. 2006).
WRKY transcription factors have been considered to be among the most important gene families in SAR (Eulgem and Somssich 2007). However, exceedingly few WRKY genes controlling either BTH-induced resistance or NPRI-mediated acquired resistance in Triticeae crops (wheat and barley) have

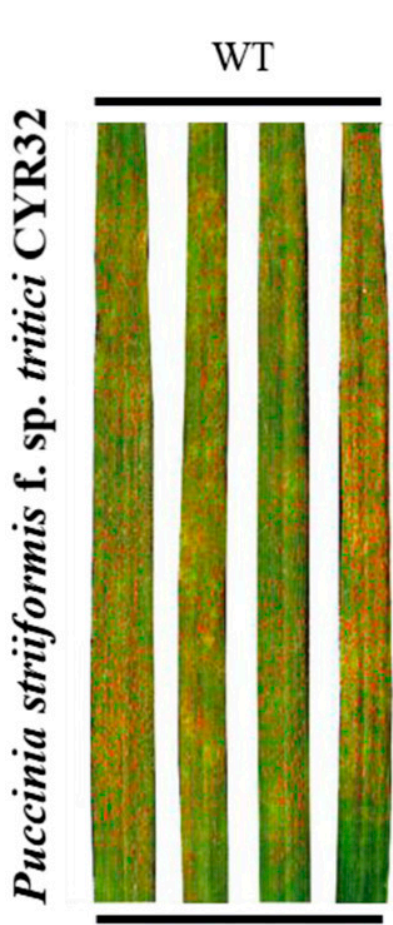

$14.0 \pm 2.9$

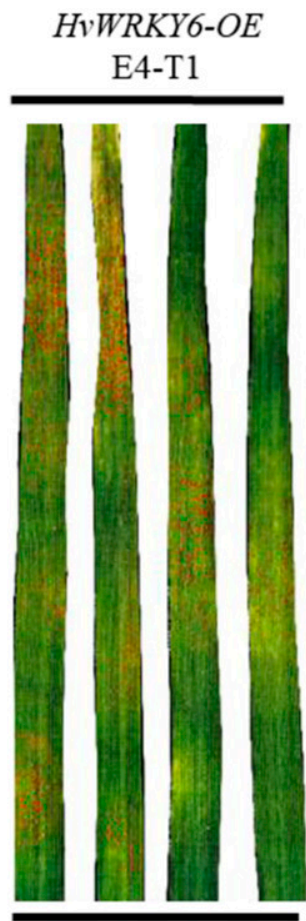

$3.1 \pm 1.2 * *$

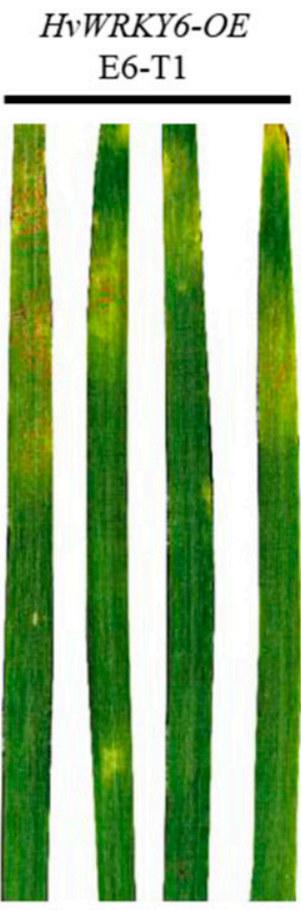

$1.4 \pm 0.7 * *$

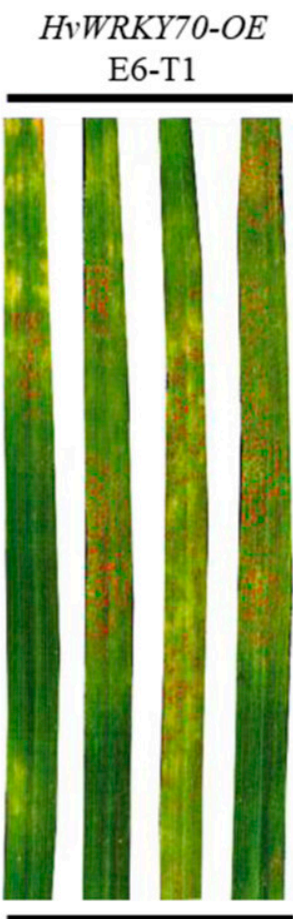

$5.6 \pm 1.3^{*}$
HvWRKY70-OE E20-T1

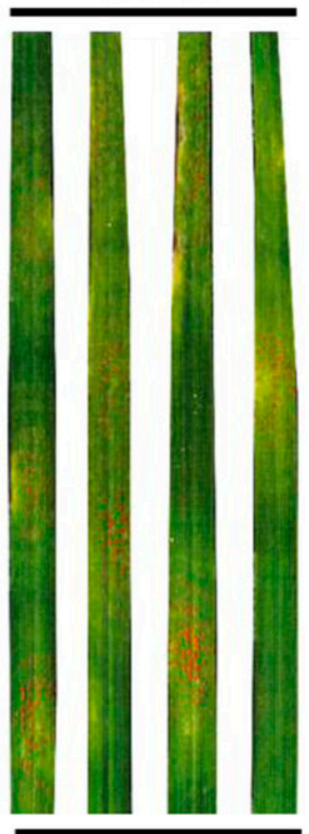

$2.7 \pm 0.7 * *$

Fig. 6. Wheat transgenic lines HvWRKY6-OE and HvWRKY7O-OE showed enhanced resistance to Puccinia striiformis f. sp. tritici. The third leaves of $H v W R K Y 6-O E$ and $H v W R K Y 70-O E$, as well as the wild-type (WT) plants, were spray inoculated with urediniospores of the highly virulent $P$. striiformis f. sp. tritici pathotype CYR32. The phenotype of the stripe rust was photographed at 15 days postinfiltration. A susceptible phenotype (P. striiformis f. sp. tritici sporulation) was exhibited in all of the genotypes. Numbers represent the average proportion of the P. striiformis f. sp. tritici sporulation area in each of the leaves. Two independent transgenic lines consisting of five to nine biological replicates were employed. Asterisks indicated the significance of the differences between the transgenic lines and the WT plants established using a Dunnett's test (* and ** indicate $P<0.05$ and 0.01 , respectively).
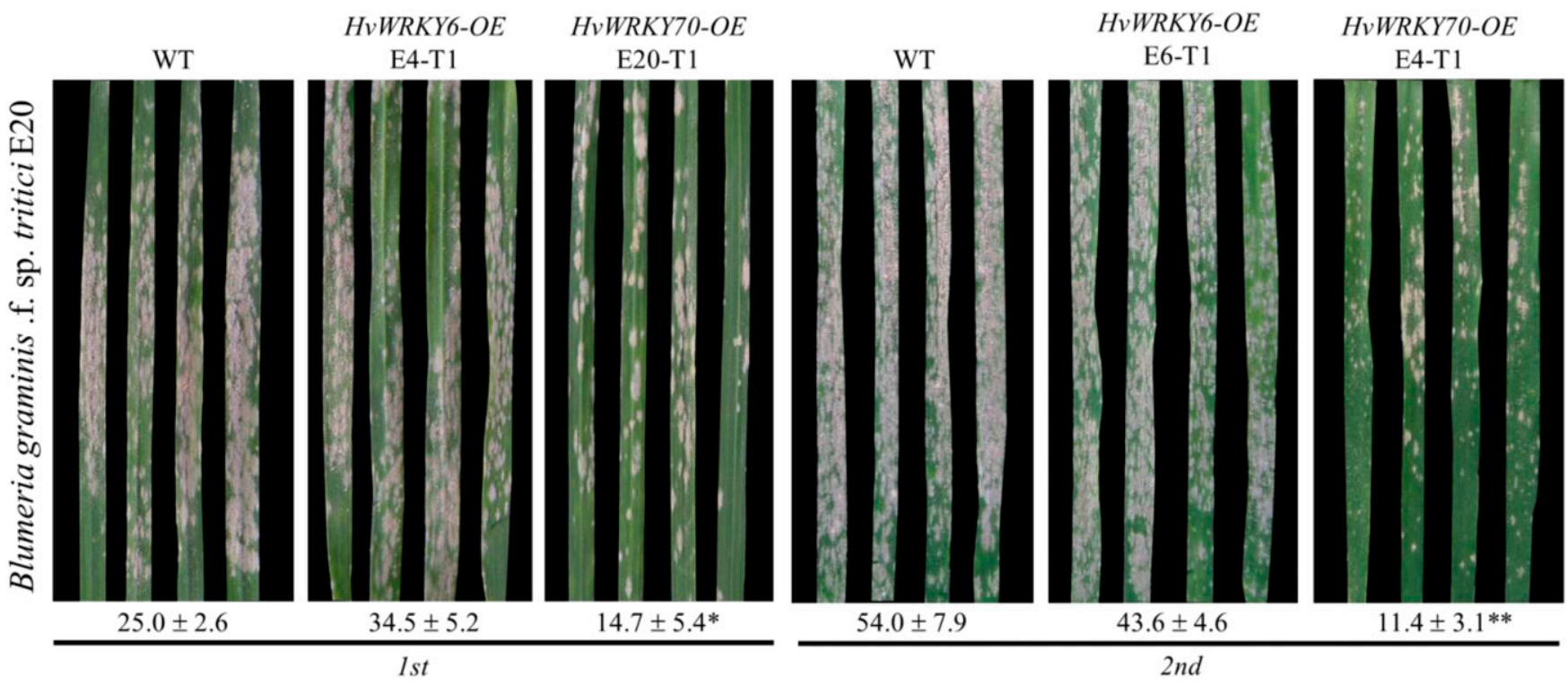

Fig. 7. Wheat transgenic line HvWRKY70-OE showed enhanced resistance to Blumeria graminis f. sp. tritici. The third leaves of the wheat transgenic lines $H v W R K Y 6-O E$ and $H v W R K Y 70-O E$, as well as the wild-type (WT) plants, were inoculated with conidia spores of the prevalent $B$. graminis f. sp. tritici isolate E20. The phenotype of the powdery mildew was photographed at 15 days postinfiltration. A susceptible phenotype (B. graminis $\mathrm{f}$. sp. tritici sporulation) was observed in all of the genotypes. Numbers represent the average proportion of the B. graminis f. sp. tritici sporulation area in each of the leaves. Two independent transgenic lines consisting of 9 to 30 biological replicates were employed. A Dunnett's test (* and ** indicate $P<0.05$ and 0.01 , respectively) was performed using SAS v9.4 software. 
been identified. In total, 46 and 171 WRKY genes were annotated in barley and wheat, respectively (Mangelsen et al. 2008; Ning et al. 2017). To explore the key transcriptional regulators of BTH-induced resistance in these two relative plant species, we profiled the expression patterns of all 46 barley WRKY genes in the transcriptome (Fig. 3). Approximately half of the $W R K Y$ genes were highly induced upon BTH treatment, indicating that a broad range of plant defense was activated by this chemical reagent. In one of our previous investigations, we profiled the regulatory network of NPRl during acquired resistance induced by $P$. syringae DC3000 using a sequencing approach similar to the one employed in the current study (Gao et al. 2018). Two barley WRKY genes, MLOC_78461 and MLOC_66134, were significantly induced by both BTH treatment and $P$. syringae DC3000 infection, with even higher expressions in barley transgenic line $w N P R 1-O E$ (Supplementary Fig. S6). We temporally designated these two genes as $H v W R K Y 6$ and $H v W R K Y 70$, respectively, based on their closest homologs in other plant species (Supplementary Fig. S7). However, because the designating system of the WRKY genes seemed to be confused, homologous analysis provided extremely limited information on the functional prediction.

Nevertheless, the generated wheat transgenic lines overexpressing HvWRKY6 and HvWRKY7O showed distinct improvements of acquired resistance, BTH-induced resistance, and resistance to biotrophic fungal pathogens of Puccinia striiformis f. sp. tritici and B. graminis f. sp. tritici. Although the specific roles of $B C I$ genes in plant defense response remain to be characterized, they have been widely considered to be downstream of the BTH treatment and involved in barley resistance to powdery mildew (Beßer et al. 2000; Jansen et al. 2005; Kogel and Langen 2005). Interestingly, all of the tested $P R$ and $B C I$ genes became more sensitive to BTH treatment only in HvWRKY70-OE (Fig. 5). Along with the observed improvement of wheat resistance to $B$. graminis $\mathrm{f}$. $\mathrm{sp}$. tritici in transgenic material (Fig. 7), we hypothesized that BTH treatment could activate the $H v W R K Y 70$ pathway, which was positively involved in resistance to powdery mildew by indirectly regulating $P R$ and $B C I$ genes. On the other hand, the basal resistance of wheat transgenic line $H v W R K Y 6-O E$ to $M$. oryzae was enhanced in association with higher expression of $P R$ genes (Fig. 4), which was similar to our previous observations of the barley transgenic line $w N P R 1-O E$ (Gao et al. 2018). We predicted that $H v W R K Y 6$ was an endogenous SA-responsive gene that acted as a partner of $N P R l$ and indirectly regulated $P R$ genes during acquired resistance. Moreover, the sporulation of $P$. striiformis f. sp. tritici was significantly diminished or delayed in both wheat transgenic lines HvWRKY6-OE and HvWRKY70-OE (Fig. 6). SA and its responsive genes are considered key components of wheat resistance to rust infections. Recent investigation showed that the endogenous SA level during wheat resistance to stripe rust was significantly elevated accompanying the hypersensitive response triggered by the $\mathrm{YrSu}$ resistance gene (Wang et al. 2017). Several $P R$ genes seemed to be shared downstream genes involved in wheat resistance to various rust diseases (e.g., Srl3-mediated high-temperature resistance to stem rust, multiple $Y r$ gene-mediated resistances to stripe rust, and the $L r 47$-mediated resistance to leaf rust) (Farrakh et al. 2018; Wu et al. 2019; Zhang et al. 2017). We speculated that wheat resistance to rust infections recruited a broad range of plant defense mechanisms, including both the $H v W R K Y 6$ and $H v W R K Y 70$-mediated regulatory pathways.

In conclusion, based on the present results and those of our previous studies, we drafted the possible regulatory networks of BTH-induced resistance and acquired resistance in wheat and barley (Fig. 8). Briefly, the Pseudomonas syringae DC3000triggered acquired resistance was controlled by NPRI with several features of SAR in model plant Arabidopsis, including elevation of endogenous SA and involvement of $P R$ genes. For the BTH-induced resistance, a large number of genes were induced by the BTH treatment in an NPRI-independent manner. Most of the $P R$ genes became sensitive to BTH treatment only in barley transgenic line $w N P R I-O E$. Overexpression of two differentially expressed $W R K Y$ genes in wheat improved wheat resistance to fungal pathogens, possibly through distinct regulatory pathways. The $H v W R K Y 70$ gene seemed to be the key regulator of $\mathrm{BTH}$-induced resistance, whereas $H v W R K Y 6$ might act as the partner of NPRl during acquired resistance.

\section{MATERIALS AND METHODS}

Plants, BTH treatment, and pathogen inoculations.

The barley transgenic lines wNPRI-OE and HvNPRl-Kd were generated in cultivar Golden Promise background in earlier investigations (Dey et al. 2014; Wang et al. 2016). The fully expanded third leaves of barley seedlings were sprayed with $0.1 \mathrm{mM}$ BTH (acibenzolar-S-methyl, CAS number 135158-54-2; Aladdin Co. Ltd., Shanghai, China). RNA samples were harvested from the BTH-sprayed leaves at $2 \mathrm{dpt}$. Wild-type plants and a mock treatment with water severed as controls. A similar protocol was applied to generate the BTHinduced resistance in wheat plants.

Seedlings of the wheat transgenic lines and wild-type plants were inoculated with stripe rust following a previous described

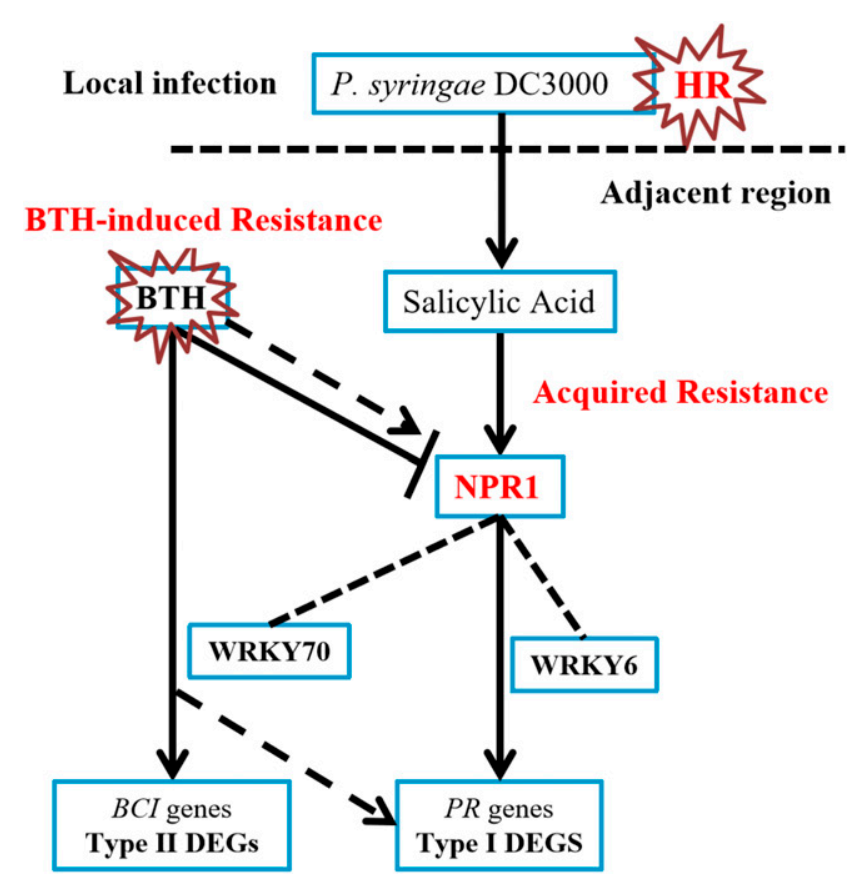

Fig. 8. Predicted regulatory network of benzothiadiazole (BTH)-induced resistance and the nonexpresser of pathogenesis-related genes 1 (NPR1)mediated acquired resistance. The Pseudomonas syringae DC3000-induced acquired resistance was controlled by NPRl with several features of systemic acquired resistance in model plant Arabidopsis, including elevation of endogenous salicylic acid and involvement of $P R$ genes. However, for the $\mathrm{BTH}$-induced resistance, large number of genes, including barley chemicalinduced $(B C I)$ genes, were induced by the BTH treatment in an NPRIindependent manner. Most of the $P R$ genes became sensitive to $\mathrm{BTH}$ treatment only in barley transgenic line overexpressing wheat $w N P R I$ ( $w N P R 1-O E)$. Overexpression of two differentially expressed $W R K Y$ genes was sufficient to improve wheat resistance to fungal pathogens, possibly through different regulatory pathways. The $H v W R K Y 70$ gene seemed to be a key regulator of BTH-induced resistance, whereas $H v W R K Y 6$ might act as a partner of $N P R 1$ during acquired resistance. $\mathrm{HR}=$ hypersensitive response and DEGs = differentially expressed genes 
procedure (Wan and Chen 2014). Briefly, fully expanded third leaves of wheat seedlings were inoculated with urediniospores of highly virulent Puccinia striiformis f. sp. tritici pathotype CYR32. The inoculated plants were maintained in relatively low-temperature conditions: $10^{\circ} \mathrm{C}$ for the first $10 \mathrm{~h}$ in the dark, followed by $16 \mathrm{~h}$ in the light at $16^{\circ} \mathrm{C}$ and $8 \mathrm{~h}$ in the dark at $8^{\circ} \mathrm{C}$. The disease symptom of stripe rust was photographed at $15 \mathrm{dpi}$. The proportion of $P$. striiformis f. sp. tritici sporulation area on each of the leaves was measured using ASSESS v2.0 software (Lamari 2008; Zhang et al. 2017). Two independent lines with five to nine biological replicates were used for each of the transgenic materials. A Dunnett's test was performed using SAS software (v9.4; SAS Institute, Cary, NC, U.S.A.).

For the powdery mildew inoculation, a prevalent $B$. graminis f. sp. tritici isolate E20 was maintained in the asexual phase in a greenhouse and used for inoculation following the procedure specified in an earlier investigation (Geng et al. 2016). Briefly, seedlings at the third-leaf stage were inoculated with conidia spores and kept in a high-humidity environment at 18 and $12^{\circ} \mathrm{C}$ (day and night, respectively) with a photoperiod of $14 \mathrm{~h}$ of light per day. The phenotype of powdery mildew was photographed at $15 \mathrm{dpi}$. The proportion of B. graminis $\mathrm{f}$. sp. tritici sporulation area on each of the leaves was measured using ASSESS software. The whole experiment was systemically repeated twice with two independent lines, and each line included 9 to 30 biological replicates. A Dunnett's test was conducted using SAS v9.4 (SAS Institute).

Acquired resistance in local leaves was induced by Pseudomonas syringae DC3000 following a previous description (Colebrook et al. 2012; Gao et al. 2018). Generally, P. syringae DC3000 was cultivated in Luria-Bertani liquid medium with rifamycin antibiotic for 2 days. The bacteria were collected by a brief centrifugation and then diluted to an optical density at $600 \mathrm{~nm}=0.5$ in distilled water. Third leaves of wheat seedlings were infiltrated with bacterial suspensions with a 1-ml needless syringe. The borders of the infiltration area were marked. Mock infiltration with distilled water served as a control. Inoculated seedlings were then transferred into a $23^{\circ} \mathrm{C}$ growth chamber. The region adjacent to the infiltration area of $P$. syringae DC3000 at 2 dpi was further used for $M$. oryzae inoculation or qRT-PCR assay.

M. oryzae isolate $\mathrm{P} 131$ was cultivated on tomato oat medium for 10 days at $25^{\circ} \mathrm{C}$ under a photoperiod of $16 \mathrm{~h}$ of light and $8 \mathrm{~h}$ of darkness. The conidia were suspended in $0.05 \%$ Tween-20 $\left(5.0 \times 10^{5}\right.$ spores $\left./ \mathrm{ml}\right)$. The region adjacent to the infiltration area (approximately $1 \mathrm{~cm}$ from the border) was press injured and further inoculated with $10 \mu \mathrm{l}$ of the M. oryzae conidia suspensions. The inoculated leaves were transferred into a growth chamber at $23^{\circ} \mathrm{C}$ and $80 \%$ humidity under a cycle of $16 \mathrm{~h}$ of light and $8 \mathrm{~h}$ of darkness. The disease symptom was photographed at 5 days postinoculation and the lesion size of $M$. oryzae on each of the leaves was measured. The whole experiment was systemically repeated twice with two independent lines of $\mathrm{HvWRKY6-}$ $O E$, and each line included six to eight biological replicates. A Dunnett's test was conducted using SAS v9.4 (SAS Institute).

\section{RNA extraction and qRT-PCR assay.}

Samples were rapidly ground into fine powders in liquid nitrogen. Total RNAs for transcriptome sequencing and qRTPCR assay were extracted using a QIAGEN plant RNA extraction kit (QIAGEN, Hilden, Germany). The first-strand cDNA was generated using a Takara reverse-transcription kit (Takara, Dalian, China). The barley elongation factor 1a (HvEFla, GenBank accession Z50789) was applied as an internal reference gene. The qRT-PCR primers for $H v P R 1 b$, $H v P R 2, H v B C I 1, H v B C I 3$, and $H v B C I 7$ were derived from our previous study (Supplementary Table S8) (Gao et al. 2018). For qRT-PCR assays performed using wheat samples, TaActin
(GenBank accession AB181991.1) was applied as an internal reference. The qRT-PCR primers for TaPRla, TaPR2, TaPR $4 b$, $T a B C I 1$, and TaBCI3 were designed and genome-specific amplification information for these wheat primers was summarized (Supplementary Table S8). Preliminary amplifications in six twofold wheat cDNA dilutions $(1: 1,1: 2,1: 4,1: 8,1: 16$, and $1: 32$ ) were utilized to determine the efficiency for each pair of the primers. Melting curves for the temperatures from 60 to $94^{\circ} \mathrm{C}$ were drawn to ensure the specificity. The threshold values (Ct) were recorded by the Roche LightCycler 96 qRT-PCR instrument (Roche, Basel, Switzerland). The relative transcript abundance was calculated following the $2^{-\Delta \mathrm{Ct}}$ method (Chen et al. 2014; Schmittgen and Livak 2008). Mean and standard error for the relative expression levels were analyzed using Microsoft Excel software (Microsoft, Redmond, WA, U.S.A.).

\section{RNA-seq and bioinformatics.}

KAPA library preparation and transcriptome sequencing were conducted by Novogene Co., Ltd. following the default protocols. The HiSeq 1000 system was utilized for the $12-\mathrm{Gb}$ sequencing for each of the samples. The Ensembl Genomes $H$. vulgare genome (The International Barley Genome Sequencing Consortium 2012) was employed for the transcriptome assembly using TopHat 2.0.8 (Trapnell et al. 2009). Then, the relative abundance for each of the transcripts assembled from mapped reads was determined by HTSeq (Trapnell et al. 2010). Comparing different groups, genes with a false discovery rate-adjusted $P$ value $<0.05$ were filtered and designated as DEGs by DESeq2 (Love et al. 2014). Enrichment of GO categories for each of the DEGs were annotated with the GOseq (Young et al. 2010). Heatmaps for the gene expression patterns were generated based on the FPKM values for each of the transcripts by $\mathrm{MeV}$ software. The neighbor-joining polygenetic tree was generated by MEGA software using protein sequences aligned using the MUSCLE method.

\section{Subcellular localization of GFP-tagged HvWRKY6 and HvWRKY70 proteins.}

The full-length open reading frames (ORF) of $H v W R K Y 6$ and $H v W R K Y 70$ genes were cloned from cDNA synthesized using RNA samples from leaves of barley cultivar Golden Promise (primers in Supplementary Table S8). They were then constructed into both pGWB5 (35S::gene-GFP) and pMDC43 (35S::GFP-gene) using Gateway LR Clonase II Enzyme (Life Technologies, Carlsbad, CA, U.S.A.). The recombinant constructs were transformed into Agrobacterium strain GV3101. Leaves from 4- to 6-week-old $N$. benthamiana plants were infiltrated with transformed Agrobacterium. An empty pGWB5 vector expressing only the GFP was used as a control. Green fluorescence was detected $48 \mathrm{~h}$ after infiltration using fluorescence microscopy (Ti-2 microscopy with fluorescent accessories; Nikon, Tokyo, Japan).

\section{Wheat transgenic lines overexpressing HvWRKYG and $H v W R K Y 70$.}

The full-length ORF of HvWRKY6 and HvWRKY70 genes were cloned into the wheat transgenic vector pLGY02 (Ubi:: gene). The recombinant vectors were then transformed into the Agrobacterium strain EHA105. Wheat transgenic lines were generated using wheat embryos of the spring common wheat cultivar JW1 by Jinan Bangdi Bio Co. Ltd., Shandong, China. Transgenic lines were initially validated by amplifying the insertion of transgene in the genomic DNA using a PCR assay (primers in Supplementary Table S8). The expression levels of $H v W R K Y 6$ and $H v W R K Y 70$ transgenes in corresponding wheat transgenic lines were determined by qRT-PCR assay (primers in Supplementary Table S8). 


\section{ACKNOWLEDGMENTS}

We thank G. Li (Shandong Academy of Agricultural Sciences, China) for excellent support in generating wheat transgenic materials.

\section{LITERATURE CITED}

Beßer, K., Jarosch, B., Langen, G., and Kogel, K.-H. 2000. Expression analysis of genes induced in barley after chemical activation reveals distinct disease resistance pathways. Mol. Plant Pathol. 1:277-286.

Cantu, D., Yang, B., Ruan, R., Li, K., Menzo, V., Fu, D., Chern, M., Ronald, P. C., and Dubcovsky, J. 2013. Comparative analysis of protein-protein interactions in the defense response of rice and wheat. BMC Genomics 14:166.

Chen, A., Li, C., Hu, W., Lau, M. Y., Lin, H., Rockwell, N. C., Martin, S. S., Jernstedt, J. A., Lagarias, J. C., and Dubcovsky, J. 2014. Phytochrome C plays a major role in the acceleration of wheat flowering under long-day photoperiod. Proc. Natl. Acad. Sci. U.S.A. 111:10037-10044.

Chern, M.-S., Fitzgerald, H. A., Yadav, R. C., Canlas, P. E., Dong, X., and Ronald, P. C. 2001. Evidence for a disease-resistance pathway in rice similar to the NPR1-mediated signaling pathway in Arabidopsis. Plant J. 27:101-113.

Colebrook, E. H., Creissen, G., McGrann, G. R., Dreos, R., Lamb, C., and Boyd, L. A. 2012. Broad-spectrum acquired resistance in barley induced by the Pseudomonas pathosystem shares transcriptional components with Arabidopsis systemic acquired resistance. Mol. Plant-Microbe Interact. 25:658-667.

Després, C., Chubak, C., Rochon, A., Clark, R., Bethune, T., Desveaux, D., and Fobert, P. R. 2003. The Arabidopsis NPR1 disease resistance protein is a novel cofactor that confers redox regulation of DNA binding activity to the basic domain/leucine zipper transcription factor TGA1. Plant Cell 15:2181-2191.

Dey, S., Wenig, M., Langen, G., Sharma, S., Kugler, K. G., Knappe, C., Hause, B., Bichlmeier, M., Babaeizad, V., Imani, J., Janzik, I., Stempfl, T., Hückelhoven, R., Kogel, K. H., Mayer, K. F., and Vlot, A. C. 2014. Bacteria-triggered systemic immunity in barley is associated with WRKY and ETHYLENE RESPONSIVE FACTORs but not with salicylic acid. Plant Physiol. 166:2133-2151.

Dong, X. 2004. NPR1, all things considered. Curr. Opin. Plant Biol. 7: 547-552.

Eulgem, T., and Somssich, I. E. 2007. Networks of WRKY transcription factors in defense signaling. Curr. Opin. Plant Biol. 10:366-371.

Farrakh, S., Wang, M., and Chen, X. 2018. Pathogenesis-related protein genes involved in race-specific all-stage resistance and non-race specific high-temperature adult-plant resistance to Puccinia striiformis f. sp. tritici in wheat. J. Integr. Agric. 17:2478-2491.

Fu, Z. Q., and Dong, X. 2013. Systemic acquired resistance: Turning local infection into global defense. Annu. Rev. Plant Biol. 64:839-863.

Gao, J., Bi, W., Li, H., Wu, J., Yu, X., Liu, D., and Wang, X. 2018. WRKY transcription factors associated with $N P R 1$-mediated acquired resistance in barley are potential resources to improve wheat resistance to Puccinia triticina. Front. Plant Sci. 9:1486.

Geng, M., Zhang, J., Peng, F., Liu, X., Lv, X., Mi, Y., Li, Y., Li, F., Xie, C., and Sun, Q. 2016. Identification and mapping of MlIW30, a novel powdery mildew resistance gene derived from wild emmer wheat. Mol. Breed. 36:130.

Görlach, J., Volrath, S., Knauf-Beiter, G., Hengy, G., Beckhove, U., Kogel, K. H., Oostendorp, M., Staub, T., Ward, E., Kessmann, H., and Ryals, J. 1996. Benzothiadiazole, a novel class of inducers of systemic acquired resistance, activates gene expression and disease resistance in wheat. Plant Cell 8:629-643.

Hafez, Y. M., Soliman, N. K., Saber, M. M., Imbabi, I. A., and Abdelaziz, A. S. 2014. Induced resistance against Puccinia triticina, the causal agent of wheat leaf rust by chemical inducers. Egypt. J. Pest Control 24:173-181.

Jansen, C., Korell, M., Eckey, C., Biedenkopf, D., and Kogel, K.-H. 2005. Identification and transcriptional analysis of powdery mildew-induced barley genes. Plant Sci. 168:373-380.

Klessig, D. F., Choi, H. W., and Dempsey, D. A. 2018. Systemic acquired resistance and salicylic acid: Past, present, and future. Mol. PlantMicrobe Interact. 31:871-888.

Kogel, K. H., and Langen, G. 2005. Induced disease resistance and gene expression in cereals. Cell. Microbiol. 7:1555-1564.

Lamari, L. 2008. Assess 2.0: Image Analysis Software for Plant Disease Quantification. American Phytopathological Society, St. Paul, MN, U.S.A.

Liu, X., Bai, X., Wang, X., and Chu, C. 2007. OsWRKY71, a rice transcription factor, is involved in rice defense response. J. Plant Physiol. 164:969-979.
Liu, X. Q., Bai, X. Q., Qian, Q., Wang, X. J., Chen, M. S., and Chu, C. C. 2005. OsWRKYO3, a rice transcriptional activator that functions in defense signaling pathway upstream of OsNPRI. Cell Res. 15:593-603.

Love, M. I., Huber, W., and Anders, S. 2014. Moderated estimation of fold change and dispersion for RNA-seq data with DESeq2. Genome Biol. 15:550.

Ma, J., Gao, X., Liu, Q., Shao, Y., Zhang, D., Jiang, L., and Li, C. 2017. Overexpression of TaWRKY146 increases drought tolerance through inducing stomatal closure in Arabidopsis thaliana. Front. Plant Sci. 8:2036.

Makandar, R., Essig, J. S., Schapaugh, M. A., Trick, H. N., and Shah, J. 2006. Genetically engineered resistance to Fusarium head blight in wheat by expression of Arabidopsis NPR1. Mol. Plant-Microbe Interact. 19:123-129.

Mangelsen, E., Kilian, J., Berendzen, K. W., Kolukisaoglu, Ü. H., Harter, K., Jansson, C., and Wanke, D. 2008. Phylogenetic and comparative gene expression analysis of barley (Hordeum vulgare) WRKY transcription factor family reveals putatively retained functions between monocots and dicots. BMC Genomics 9:194.

Molina, A., Görlach, J., Volrath, S., and Ryals, J. 1999. Wheat genes encoding two types of PR-1 proteins are pathogen inducible, but do not respond to activators of systemic acquired resistance. Mol. PlantMicrobe Interact. 12:53-58.

Nakayama, A., Fukushima, S., Goto, S., Matsushita, A., Shimono, M., Sugano, S., Jiang, C. J., Akagi, A., Yamazaki, M., Inoue, H., and Takatsuji, H. 2013. Genome-wide identification of WRKY45-regulated genes that mediate benzothiadiazole-induced defense responses in rice. BMC Plant Biol. 13:150

Ning, P., Liu, C., Kang, J., and Lv, J. 2017. Genome-wide analysis of WRKY transcription factors in wheat (Triticum aestivum L.) and differential expression under water deficit condition. PeerJ 5:e3232.

Schmittgen, T. D., and Livak, K. J. 2008. Analyzing real-time PCR data by the comparative C(T) method. Nat. Protoc. 3:1101-1108.

Shimono, M., Sugano, S., Nakayama, A., Jiang, C. J., Ono, K., Toki, S., and Takatsuji, H. 2007. Rice WRKY45 plays a crucial role in benzothiadiazole-inducible blast resistance. Plant Cell 19:2064-2076.

The International Barley Genome Sequencing Consortium. 2012. A physical, genetic and functional sequence assembly of the barley genome. Nature 491:711-716.

Trapnell, C., Pachter, L., and Salzberg, S. L. 2009. TopHat: Discovering splice junctions with RNA-Seq. Bioinformatics 25:1105-1111.

Trapnell, C., Williams, B. A., Pertea, G., Mortazavi, A., Kwan, G., van Baren, M. J., Salzberg, S. L., Wold, B. J., and Pachter, L. 2010. Transcript assembly and quantification by RNA-Seq reveals unannotated transcripts and isoform switching during cell differentiation. Nat. Biotechnol. 28:511-515.

Vallélian-Bindschedler, L., Métraux, J. P., and Schweizer, P. 1998. Salicylic acid accumulation in barley is pathogen specific but not required for defense-gene activation. Mol. Plant-Microbe Interact. 11:702-705.

Wan, A., and Chen, X. 2014. Virulence characterization of Puccinia striiformis f. sp. tritici using a new set of Yr single-gene line differentials in the United States in 2010. Plant Dis. 98:1534-1542.

Wang, D., Amornsiripanitch, N., and Dong, X. 2006. A genomic approach to identify regulatory nodes in the transcriptional network of systemic acquired resistance in plants. PLoS Pathog. 2:e123.

Wang, X., Bi, W., Gao, J., Yu, X., Wang, H., and Liu, D. 2018. Systemic acquired resistance, $N P R 1$, and pathogenesis-related genes in wheat and barley. J. Integr. Agric. 17:2468-2477.

Wang, X., Wang, Y., Liu, P., Ding, Y., Mu, X., Liu, X., Wang, X., Zhao, M., Huai, B., Huang, L., and Kang, Z. 2017. TaRarl Is Involved in Wheat Defense against Stripe Rust Pathogen Mediated by $\mathrm{YrSu}$. Front. Plant Sci. 8:156.

Wang, X., Yang, B., Li, K., Kang, Z., Cantu, D., and Dubcovsky, J. 2016. A conserved Puccinia striiformis protein interacts with wheat NPR1 and reduces induction of pathogenesis-related genes in response to pathogens. Mol. Plant-Microbe Interact. 29:977-989.

Wu, J., Gao, J., Bi, W., Zhao, J., Yu, X., Li, Z., Liu, D., Liu, B., and Wang, X. 2019. Genome-wide expression profiling of genes associated with the Lr47-mediated wheat resistance to leaf rust (Puccinia triticina). Int J. Mol. Sci. 20:4498.

Young, M. D., Wakefield, M. J., Smyth, G. K., and Oshlack, A. 2010. Gene ontology analysis for RNA-seq: Accounting for selection bias. Genome Biol. 11:R14.

Zhang, W., Chen, S., Abate, Z., Nirmala, J., Rouse, M. N., and Dubcovsky, J. 2017. Identification and characterization of $\mathrm{Sr} 13$, a tetraploid wheat gene that confers resistance to the Ug99 stem rust race group. Proc. Natl. Acad. Sci. U.S.A. 114:E9483-E9492. 